\title{
Zur Kenntnis einiger physikalischer Eigenschaften von Elementen und einfachen Verbindungen.
}

\author{
Von W. Herz.
}

Die Systematik der Eigenschaften von Elementen und Verbindungen beruht immex auf dem periodischen System. So wunderbar die Klassifizierung des Verhaltens der Stoffe im allgemeinen nach diesem Prinzip gelingt, so ist doch andererseits die Tatsache bemerkenswert, daß bei fast allen Eigenschaften gewisse einzelne Unstimmigkeiten in der Reihenfolge auftreten, und daß das Steigen oder Fallen der Eigenschaftswerte in verschiedenen Gruppen des Systems unregelmäßig wechselt. Am meisten studiert sind diese Störungen bei den Atomgewichten selbst, und über die Umkehrung mancher Atomgewichte wie Jod-Tellur oder Argon-Kalium hat sich eine ganze Literatur gebildet. Erst durch die auf der Entwicklung der Radioaktivität begründete Auffassung über die isotopischen Elemente oder Elementplejaden - vornehmlich durch FaJANS und Sodpy - ist diese letztere Erscheinung unserem Verständnis näher gerückt worden. Inwieweit andere Differenzen bei physikalischen Eigenschaften auch auf die komplexe Natur der Elemente zurückgeführt werden können, ist noch wenig diskutiert; im allgemeinen sind ja die Eigenschaften der Komponenten einer Plejade so übereinstimmend, daß Unterschiede nicht auftreten, doch werden vielleicht bei manchen Qualitäten, die direkt von der Masse abhängen, Abweichungen nachweisbar werden. ${ }^{\mathbf{1}}$ Für die Betrachtung der Eigenschaftsunstimmigkeiten im Sinne des periodischen Systems sind weiterhin wahrscheinlich, wenigstens soweit feste Elemente in Betracht kommen, auch die zahlreichen Untersuchungen über die Metastabilität der Metalle von größter Bedeutung, die E. CoHen mit seinen Schülern ausgeführt hat und die beweisen, daß das Auftreten mehrerer Modifikationen viel häufiger ist als man früher annahm.

Wenn auch die Tatsache gewisser Unregelmäßigkeiten im periodischen System längst wohl bekannt ist, so erscheint es viel-

${ }^{1}$ K. FaJans, Zeitschr. Elektrochem. 20, 459.

Z. anorg. u. allg. Chem. Bd. 94. 
leicht nicht ganz unnötig, eine Reihe solcher Erscheinungen einmal zusammenhängend darzustellen, da - soweit ich weiß - in dell üblichen Lehrbüchern auf diese Verhältnisse nur ganz allgemein kurz hingewiesen wird, und selbst das tüchtige Buch von Rudorf ${ }^{1}$ über das periodische System ist für diese lälle nicht ausführlich genug. Außerdem lassen sich bei dieser Behandlung eine ganze Anzahl von Berechnungen angeben, die wenigstens zum T'eil gerade für Elemente und einfache Verbindungen noch nicht bekannt sind, während ein anderer l'eil der ki itischen Nachprüfung bedarf.

Zucrst will ich mit der Aufzählung der normalen Siedepunkte bei $760 \mathrm{~mm}$ J)ruck beginnen; im nachfolgenden stehen unter $t$ die Temperaturen in gewöhnlicher und unter $T$ die Werte in absoluter Zählung.

\begin{tabular}{|c|c|c|c|c|c|c|c|c|}
\hline \multicolumn{3}{|c|}{ Nullte Gruppe } & \multicolumn{3}{|c|}{ Erste Ciruppe } & \multicolumn{3}{|c|}{ Zweite Gruppe } \\
\hline & $t$ & $T$ & & . & $T^{\prime}$ & & $t$ & $T$ \\
\hline $\mathrm{He}$ & $-268.7 \mathrm{I}$ & 4.29 & $\mathrm{Li}$ & 1400 & 1673 & $\mathrm{Zn}$ & 929.6 & 1202.6 \\
\hline $\mathrm{Ar}$ & -186.1 & 86.9 & $\mathrm{Na}$ & 877.5 & 1150.5 & Cd & 778 & 1051 \\
\hline $\mathrm{Kr}$ & -151.7 & 121.3 & $\mathrm{~K}$ & 757.5 & 1030.5 & - $\mathrm{Hg}$ & 357.25 & 630.25 \\
\hline 7 & -109.1 & 163.9 & $\mathrm{Rb}$ & 696 & 969 & & & \\
\hline & & & $\mathrm{Cs}_{3}$ & 670 & 943 & & & \\
\hline
\end{tabular}

Vierte Gruppe

$\begin{array}{ccc} & t & T \\ \mathrm{Sn} & 2270 & 2543 \\ \mathrm{~Pb} & 1525 & 1798\end{array}$

$\begin{array}{ccc} & \text { Fünfte } & \text { Gruppe } \\ & t & T \\ \mathrm{~N} & -195.67 & 77.33 \\ \mathrm{P} & 287.3 & 560.3 \\ \text { As } & 616 & 889 \\ \text { Sb } & 1440 & 1713 \\ \text { Bi } & 1420 & 1693\end{array}$

\begin{tabular}{lcc}
\multicolumn{3}{c}{ Sechste Gruppe } \\
& $t$ & $T$ \\
$O$ & -182.8 & 90.2 \\
$S$ & 445 & 718 \\
Se & 690 & 963 \\
Te & 1390 & 1663
\end{tabular}

Siebente Gruppe

\begin{tabular}{lcl} 
& \multicolumn{1}{c}{$\ell$} & $T$ \\
$\mathrm{~F}$ & -187 & \multicolumn{1}{c}{86} \\
$\mathrm{Cl}$ & -33.6 & $\mathbf{2 3 9 . 4}$ \\
$\mathrm{Br}$ & 63 & 336 \\
$\mathrm{~J}$ & 184.35 & 457.35
\end{tabular}

Die Betrachtung dieser Zahlen lehrt, daß in jeder Gruppe entweder regelmäßiges Steigen oder Fallen der Siedepunkte stattfindet, mit einziger Ausnahme des Antimons, dessen Siedepunkt nach der neuesten Angabe von Gresnwood ${ }^{3}$ zwischen den Siedepunkten von Arsen und Wismut liegen soll. Man findet, daß in den Gruppen der Metalloide (nullte, fünfte, sechste, siebente Gruppe) die Siedepunkte mit wachsendem Atomgewicht steigen und bei den Gruppen der Metalle (erste, zweite, vierte Gruppe) ebenso sinken. Vielleicht - falls nicht etwa Versuchsfehler vorliegen -- beruht auf diesem Verhalten die ebenerwähnte Anomalie Antimon-Wismut; bei den

1 Hamburg und Leipzig 1904.

2 Bei diesem Siedepunkte fehlt die Angabe des Druckes.

3 Proc. Roy. Soc., Ser. A. 92, 396. 
vier ersten Gliedern hat man gemäß der Metalloidnatur Zunahme der Siedepunkte; das stärkst metallische Glied der Reihe, das Wismut, zeigt entsprechend den anderen Metallfamilien ein Absinken.

Die Siedepunkte von Kupfer, Silber, Gold sind auch noch in der Literatur angegeben; ich habe sie aber hier nicht verwertet, da die von verschiedenen Autoren gemachten Siedepunktsangaben untereinander so große Differenzen aufweisen, daß bei den an und für sich für die drei Metalle nicht sehr erheblichen Unterschieden ein Vergleich unmöglich scheint.

Sollen die Siedepunkte der Elemente in der Weise nebeneinandergestellt werden, wie die Atomgewichte aufeinanderfolgen, so reichen die angeführten Zahlen nur zu einer Veranschaulichung der auf der rechten und linken Seite des Systems stehenden Grundstoffe aus, während die Mittelglieder fehlen, und man hat (Siedepunkte in absoluter Zählung)

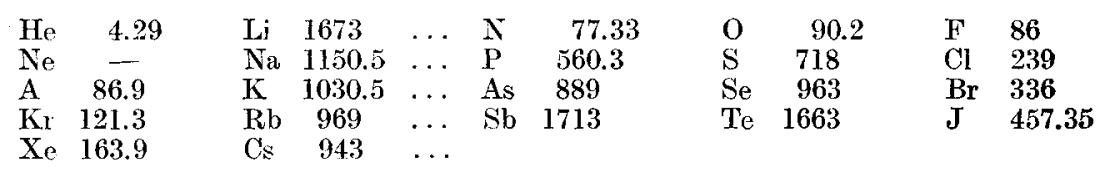

Von links ausgehend steigen die Siedepunkte und erreichen wahrscheinlich in der Mitte ihr Maximum, wo die Elemente Bor, Kohlenstoff, Silicium, Titan usw. stehen, deren Siedetemperaturen nicht bestimmt sind; dann fallen die Siedepunkte wieder. Vergleichen wir jedoch die Siedepunkte der Stickstoff-Sauerstoff-Fluorreihen, so ist eine regelmäßige Periodizität nicht zu erkennen.

Außer den Siedepunkten bei Atmosphärendruck sind auch bei manchen Elementen noch die Siedepunkte bei anderen Drucken bekannt, und daraus läßt sich die Regel von RAMSAY berechnen, wonach das Verhältnis der zu gleichen Drucken gehörigen Siedepunkte in absoluter Zählung für analoge Stoffe konstant sein soll. Die annähernde Gültigkeit dieser Beziehung für einzelne Gruppen des Systems erweist die nachfolgende Übersicht:

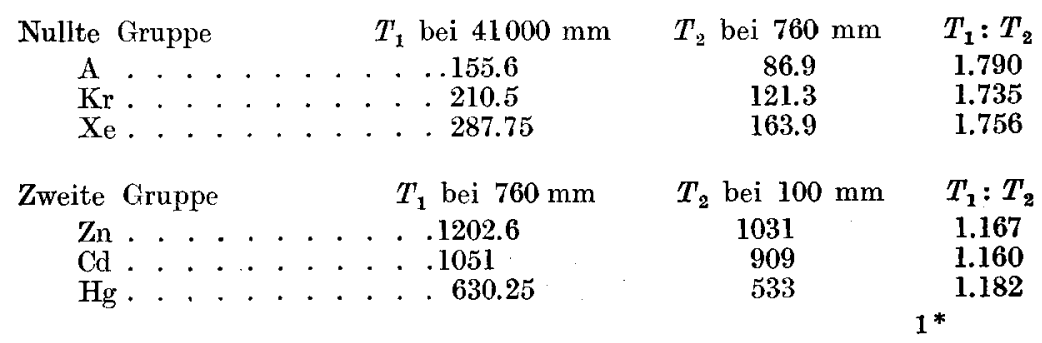


Vierte Gruppe

$$
\begin{aligned}
& \mathrm{Sn} . . . . . . .2543 \\
& \mathrm{~Pb} \text {. . . . . . } 1798
\end{aligned}
$$
$2373 \quad 1.071$
$1693 \quad 1.062$

Fünfte Gruppe

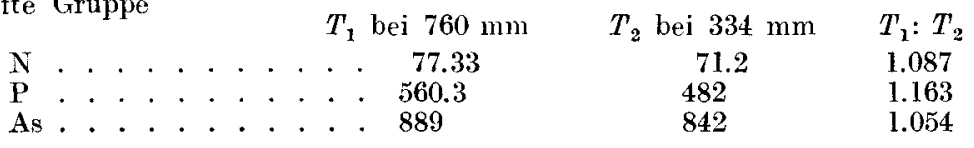

Sechste Gruppe

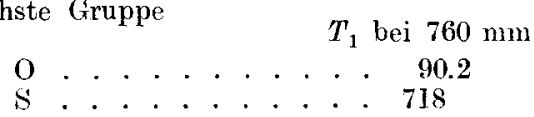
$T_{2}$ bei $250 \mathrm{~mm} \quad T_{1}: T_{2}$ $81 \quad 1.113$
$652.4 \quad 1.100$

Siebente Gruppe

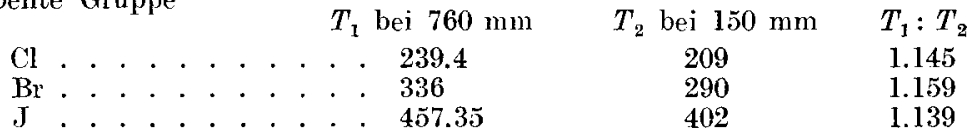

SiedepunktsregelmäBigkeiten müssen ebenso wie bei den Elementen auch bei analogen Verbindungen erscheinen; in meiner Auswahl habe ich mich der Übersichtlichkeit wegen auf die Wiedergabe der Siedetemperaturen in gewöhnlicher Zählung beschränkt.

Erste Gruppe

$$
\begin{array}{ll}
\mathrm{Cu}_{2} \mathrm{Cl}_{2} & 954-1032 \\
\mathrm{Cu}_{2} \mathrm{Br}_{2} & 861-954 \\
\mathrm{Cu}_{2} \mathrm{~J}_{2} & 751--772
\end{array}
$$

Zweite Gruppe

$$
\begin{aligned}
& \mathrm{CaBr}, \quad 806-812 \\
& \mathrm{CaJ}_{2} \quad 708-719
\end{aligned}
$$

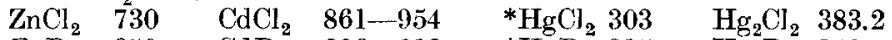

$$
\begin{aligned}
& \mathrm{ZnBr}_{2} 650 \quad \mathrm{CdBr}_{2} \quad 806-812 \quad{ }^{*} \mathrm{HgBr}_{2} 315 \quad \mathrm{Hg}_{2} \mathrm{Br}_{2} 340-350
\end{aligned}
$$

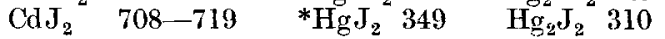

Dritte Gruppe

$$
\text { Vierte Gruppe }
$$$$
\mathrm{BF}_{3}-101
$$$$
\mathrm{BCl}_{3} \quad 17
$$$$
\begin{array}{llll}
\mathrm{BBr}_{3} & 90.5 & \mathrm{AlBr}_{3} & 260
\end{array}
$$$$
\begin{array}{llll}
\mathrm{BJ}_{3} & 210 & \mathrm{AlJ}_{3} & 350
\end{array}
$$

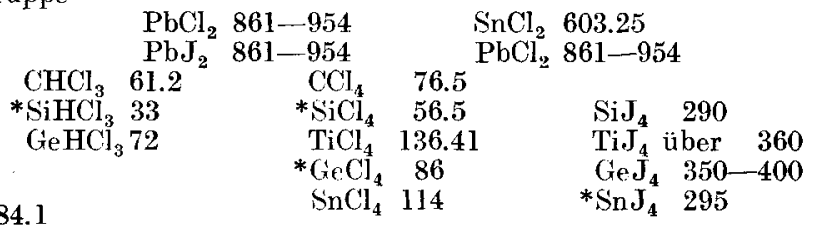

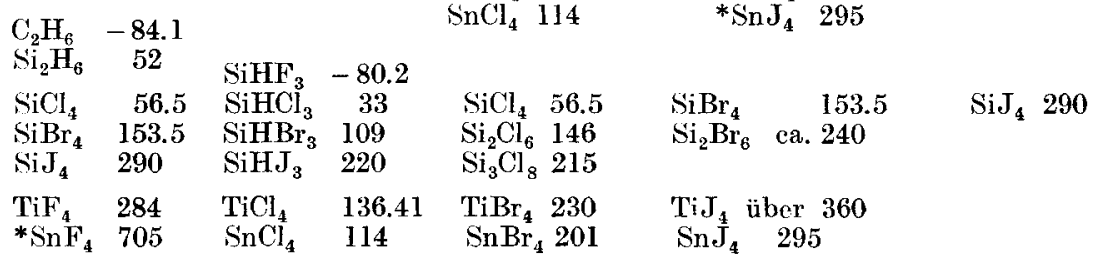




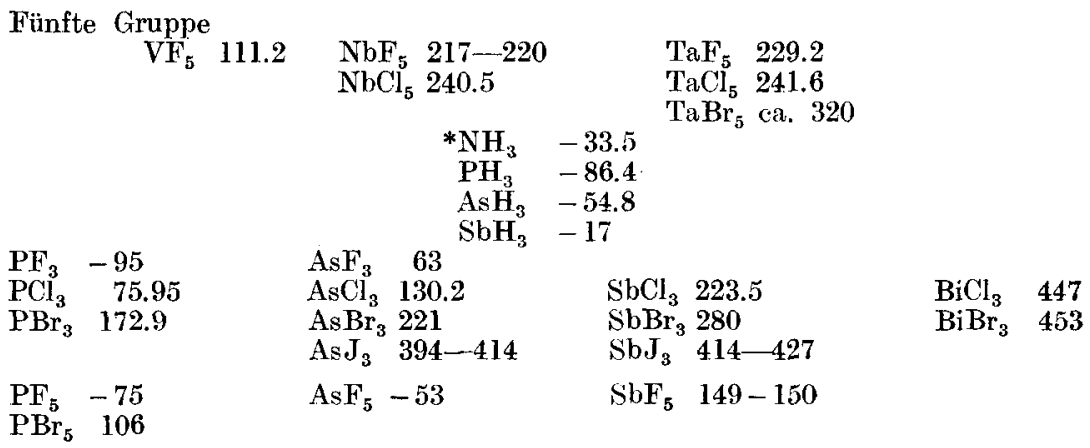

Sechste Gruppe

\begin{tabular}{|c|c|c|c|c|c|}
\hline $\begin{array}{l}{ }^{*} \mathrm{OH}_{2} \\
\mathrm{SH}_{2} \\
\mathrm{SeH}_{2} \\
\mathrm{TeH}_{2}\end{array}$ & $\begin{array}{c}100 \\
-61.8 \\
-41 \\
0\end{array}$ & $\begin{array}{l}\mathrm{O}_{2} \mathrm{C} \\
\mathrm{S}_{2} \mathrm{C}\end{array}$ & $\begin{array}{l}-79 \\
46.25\end{array}$ & & \\
\hline $\begin{array}{l}\mathrm{SOCl}_{2} \\
\mathrm{SeOCl}_{2}\end{array}$ & $\begin{array}{r}78.8 \\
179.5\end{array}$ & & $\begin{array}{lr}\mathrm{SCl}_{2} & 59 \\
\mathrm{TeCl}_{2} & 324\end{array}$ & $\underset{\mathrm{CrO}_{2} \mathrm{Cl}_{2}}{\mathrm{SO}_{2} \mathrm{Cl}_{2}}$ & $\begin{array}{r}69.1 \\
115.9\end{array}$ \\
\hline $\begin{array}{l}{ }^{*} \mathrm{MoF}_{6} \\
\mathrm{WF}_{6} \\
\mathrm{UrF}_{6}\end{array}$ & $\begin{array}{l}35 \\
19.5 \\
56\end{array}$ & & $\begin{array}{ll}\mathrm{MoCl}_{5} & 268 \\
\mathrm{WCl}_{5} & 275.6\end{array}$ & $\underset{\mathrm{WOF}_{4}}{\mathrm{MoOF}_{4}}$ & $\begin{array}{l}180 \\
185-190\end{array}$ \\
\hline
\end{tabular}

Siebente Gruppe

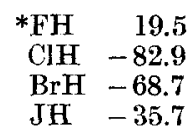

Diese Zahlen erweisen, daß bei den Siedepunkten homologer Verbindungen zahlreiche Regelmäßigkeiten auftreten, die sich in der hier gewählten Schreibweise sowohl von oben nach unten als auch von links nach rechts verfolgen lassen; doch sind allgemeine Gesetzlichkeiten nicht angebbar. So können die Siedepunkte verschiedener Haloide eines Elements mit wachsendem Atomgewicht des Halogens steigen oder fallen, und andererseits, während $\mathrm{SnF}_{4}$ einen höheren Kochpunkt als $\mathrm{TiF}_{4}$ besitzt, liegt der Siedepunkt von $\mathrm{SnCl}_{4}$ niedriger als der von $\mathrm{TiCl}_{4}$; sogar die Reihenfolge der Siedepunkte von Merkuro- und Merkurihaloiden ist umgekehrt. Außerdem erscheinen innerhalb verschiedener Gruppen einzelne Unstetigkeiten, indem die Siedepunkte mancher Verbindungen herausfallen, was durch Sternchen an den Formeln angedeutet ist. Besonders auffällig ist die Siedepunktsanomalie bei $\mathrm{HF}$ und $\mathrm{H}_{2} \mathrm{O}$, was man bekanntlich durch Polymerisation dieser Flüssigkeiten im Gegensatz zu ihren Homologen zu erklären versucht hat. Inwieweit andere ähnliche Anomalien eine entsprechende Auffassung zulassen, ist nicht bekannt. 
Auch für einige der hier erwähnten Verbindungen läßt sich die Ramsaysche Regel berechnen und ihre Gültigkeit feststellen:

Zweite Gruppe

\begin{tabular}{|c|c|c|c|}
\hline $\mathrm{HgCl}_{2}$ & $\begin{array}{c}T_{1} \text { bei } 760 \mathrm{~mm} \\
576\end{array}$ & $T_{2}$ bei $3 \mathrm{~mm}$ & $\begin{array}{c}T_{1}: T_{2} \\
1.355\end{array}$ \\
\hline $\mathrm{HgBr}_{2}$ & 588 & 435 & 1.351 \\
\hline $\mathrm{HgJ}_{2}$ & 622 & 450 & 1.382 \\
\hline
\end{tabular}

Vierte Gruppe

\begin{tabular}{|c|c|c|c|c|}
\hline $1+$ & & $T_{1}$ bei $760 \mathrm{~mm}$ & $T_{2}$ bei $50 \mathrm{~mm}$ & $T_{1}: T_{2}$ \\
\hline & & . . 349.5 & 280.5 & 1.245 \\
\hline (4) & & 329.5 & 264 & 1.248 \\
\hline $\mathrm{GeCl}_{4}$ & . & · . 359 & 284 & 1.264 \\
\hline & . & 387 & 312.6 & 1.238 \\
\hline
\end{tabular}

Fünfte Gruppe

$$
\begin{aligned}
& T_{1} \text { bei } 760 \mathrm{~mm} \\
& \mathrm{NH}_{3} \text {. . . . } 239.5 \\
& \mathrm{PH}_{3} \text {. . . . . . } 186.6 \\
& \begin{array}{cc}
T_{2} \text { bei } 235 \mathrm{~mm} & T_{1}: T_{2} \\
219 & 1.093 \\
167 & 1.118
\end{array}
\end{aligned}
$$

Sechste Gruppe

$$
\begin{aligned}
& T_{1} \text { bei } 760 \mathrm{~mm} \\
& \mathrm{OH}_{2} \text {. . . . . } 373 \\
& \mathrm{SH}_{2} \text {. . . . } 211.2 \\
& \mathrm{SeH}_{2} \text {. . . . } 232 \\
& T_{1} \text { bei } 760 \mathrm{~mm} \\
& \mathrm{O}_{2} \mathrm{C} \ldots \ldots . . \quad 194.8 \\
& \mathrm{~S}_{2} \mathrm{C} \text {. . . . } 319.2
\end{aligned}
$$

Siebente Gruppe

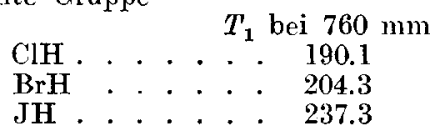

$$
\begin{array}{cc}
T_{2} \text { bei } 9120 \mathrm{~mm} & T_{1}: T_{2} \\
461.6 & 0.808 \\
278 & 0.760 \\
303.8 & 0.760 \\
T_{2} \text { bei } 42.5 \mathrm{~mm} & T_{1}: T_{2} \\
163 & 1.195 \\
253 & 1.261
\end{array}
$$

\begin{tabular}{|c|c|c|c|c|}
\hline & \multicolumn{4}{|c|}{ Elemente: } \\
\hline te Gruppe & $t$ & $T_{k}$ & $T_{s}: T_{k}$ & $p_{k}$ \\
\hline $\mathrm{He}$ & $>-268$ & $>5$ & - & $2-3$ \\
\hline $\mathrm{Ne}$. & $<-205$ & $<68$ & - & 29 \\
\hline A & . -117.4 & 155.6 & 0.56 & 52.9 \\
\hline $\mathbf{K r}$ & . -62.5 & 210.5 & 0.58 & 54.3 \\
\hline $\mathrm{Xe}$ & . $\quad 14.7$ & 287.7 & 0.58 & 57.2 \\
\hline
\end{tabular}

$\begin{array}{cc}T_{2} \text { bei } 138 \mathrm{~mm} & T_{1}: T_{2} \\ 163 & 1.164 \\ 172 & 1.186 \\ 204.5 & 1.159\end{array}$

Nach GULdBerg ${ }^{1}$ soll der Siedepunkt in absoluter Zählung etwa zwei Drittel von der kritischen Temperatur betragen. Danach müssen dieselben Beziehungen wie bei den Siedepunkten auch bei den kritischen Temperaturen erscheinen. In der folgenden Tabelle stehen unter $t_{k}$ die kxitischen Temperaturen in gewöhnlicher Zählung, unter $T_{k}$ die entsprechenden absoluten Werte und unter $T_{s}: T_{k}$ ( $T_{s}$ gleich Siedetemperaturen in absoluter Zählung) die Quotienten, welche nach GGLDBERG zwei Drittel betragen sollen. In zwei Beispielen konnten auch die kritischen Drucke unter $p_{k}$ beigefügt werden.

1 Zeitschr. phys. Chem. 5, 374. 


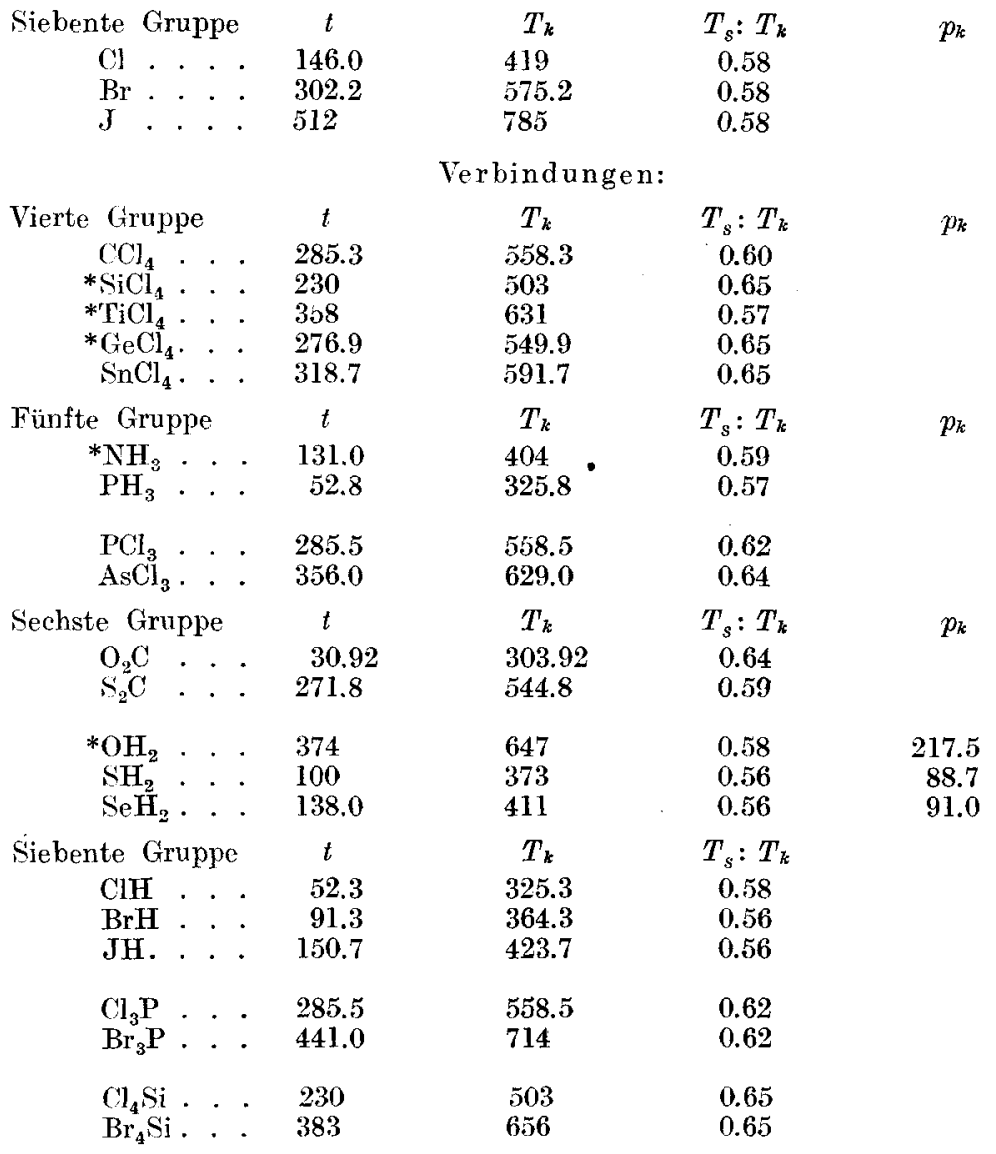

Die Zahlen unter $T_{s}: T_{k}$ beweisen die annähernde Richtigkeit der GuLdBenGschen Regel, die bei genauem Zutreffen 0.66 liefern müßte; in Wirklichkeit sind die Quotienten etwas kleiner.

Innerhalb jeder Gruppe steigen im allgemeinen die kritischen Temperaturen mit wachsendem Atomgewicht; nur bei $\mathrm{NH}_{3}$ ist die kritische Temperatur höher als bei $\mathrm{PH}_{3}$. Das $\mathrm{H}_{2} \mathrm{O}$ fällt mit seiner kritischen Temperatur aus der Reihe, und ebenso zeigen sich Unstetigkeiten bei den Chlorverbindungen der vierten Gruppe, was ganz entsprechend auch bei den Siedepunkten zu beobachten war. Die Reihenfolgen der kritischen und Siedetemperaturen sind gleich.

In den beiden Fällen, wo die kritischen Drucke angebbar waren, wachsen diese mit größerem Atomgewicht; nur das Wasser bildet eine Ausnahme.

In naher Beziehung zu den Siedetemperaturen stehen die Ver- 
dampfungswärmen; doch ist das hier vorhandene Material leider sehr gering, so daß ich nur wenige Zahlen anführen kann ( $\lambda$ Verdampfungswärme in Kalorien für $1 \mathrm{~kg}$ Substanz):

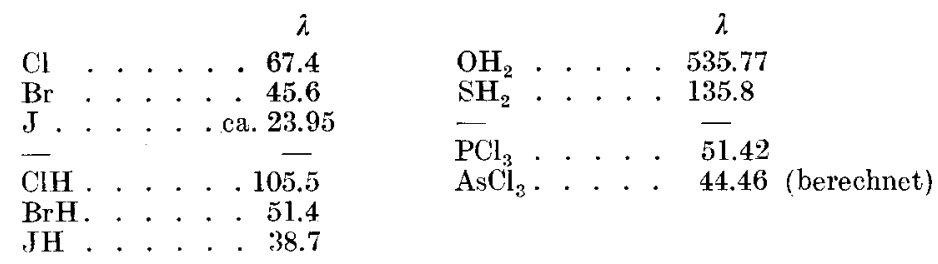

Die Verdampfungswärmen fallen mit steigendem Atomgewicht. Da bekanntlich die Verdampfungswärmen den molekularen Siedepunktserhöhungen $E$ nach der Gleichung

$$
E=\frac{R T^{2}}{\lambda}
$$

umgekehrt proportional sind, so müssen die ebullioskopischen Konstanten mit dem Atomgewicht steigen.

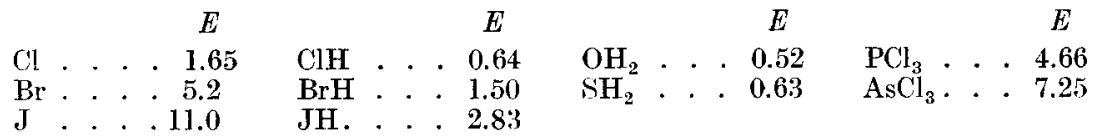

Für diese Elemente und einfachen Verbindungen läßt sich die Regel von Trouton berechnen, wonach die molekulare Verdampfungswärme $\lambda M$ dividiert durch die Siedetemperatur in absoluter Zählung $T$ konstant (gleich $20-22$ ) sein soll; die von Nernst revidierte Formel

$$
\frac{\lambda M}{T}=9.5 \log T-0.007 T
$$

\begin{tabular}{|c|c|c|c|c|c|c|}
\hline & & $\lambda$ & $M$ & $T$ & $M \hat{\lambda}: T$ & $9.5 \log T-0.007 T$ \\
\hline $\begin{array}{l}\mathrm{Cl} . \\
\mathrm{Br}\end{array}$ & $\therefore$ & $\begin{array}{l}67.4 \\
45.6\end{array}$ & $\begin{array}{r}70.92 \\
159.84\end{array}$ & $\begin{array}{l}239.4 \\
336\end{array}$ & $\begin{array}{r}20.0 \\
21.69\end{array}$ & $\begin{array}{l}20.9 \\
21.65\end{array}$ \\
\hline $\begin{array}{l}\mathrm{ClH} . \\
\mathrm{BrH} . \\
\text { JH }\end{array}$ & $\therefore$ & $\begin{array}{r}105.5 \\
51.4 \\
38.7\end{array}$ & $\begin{array}{r}36.47 \\
80.93 \\
127.93\end{array}$ & $\begin{array}{l}190.1 \\
204.3 \\
237.3\end{array}$ & $\begin{array}{l}20.24 \\
20.36 \\
20.86\end{array}$ & $\begin{array}{l}20.32 \\
20.52 \\
20.90\end{array}$ \\
\hline $\begin{array}{l}{ }^{*} \mathrm{OH}_{2} \\
\mathrm{SH}_{2} .\end{array}$ & $\therefore$ & $\begin{array}{l}535.77 \\
135.8\end{array}$ & $\begin{array}{l}18.016 \\
34.09\end{array}$ & $\begin{array}{l}373 \\
211.2\end{array}$ & $\begin{array}{l}25.89 \\
21.92\end{array}$ & $\begin{array}{l}21.82 \\
20.60\end{array}$ \\
\hline $\mathrm{PCl}_{3}$. & . . & 51.42 & 137.42 & 348.95 & 20.24 & 21.71 \\
\hline
\end{tabular}

schließt sich den Versuchen gut an.

Innèrhalb jeder Gruppe des periodischen Systems steigt der Troutonsche Quotient mit dem Atomgewicht, entsprechend dem Wachsen der Siedetemperaturen, worauf von NERNsT ${ }^{1}$ eingehend

1 Gött. Nachr. 1906, Heft 1. 
aufmerksam gemacht worden ist; nur das Wasser mit seinem abnormen Siedepunkte und seinem Polymerisationszustande fällt heraus. Die anderen Flüssigkeiten sind nicht assoziiert.

Zahlreich sind die Angaben über die Schmelzpunkte der Elemente, die ich unter $t$ in gewöhnlicher und unter $T$ in absoluter Zählung anführe.

Nullte Gruppe

$$
\begin{aligned}
& \begin{array}{ccc}
t & T
\end{array} \\
& \text { A. . . . } \quad-187.9 \quad 85.1 \\
& \mathrm{Kr} . . . . \quad-169 \quad 104 \\
& \mathrm{Xe} . . . \quad-140 \quad 133
\end{aligned}
$$

Erste Gruppe $t \quad T$
a) $\mathrm{Li} . . .186$
$\mathrm{Na} . . .97 .5$
$\mathrm{K} . .62 .5$
$\mathrm{Rb}$. . . 38.5
$\mathrm{Cs} . .26 .5$
335.5
311.5
299.5
b) $\mathrm{Cu} . \ldots 1084.1$
*Ag . . . . 961.5
Au . . . 1064
$T$
1357.1
1234.5
1337.1

$T$

692.4

594.7

233.56

Zweite Gruppe $t \quad T$

a) ${ }^{*} \mathrm{Be} . .>960 \quad>1233$

$\mathrm{Mg}$. . 650

923

Ca (unrein) 780

1053

Sr. . . ca. 800

1073

$\mathrm{Ba}$. . 850

1123

Dritte Gruppe $t \quad T$

$T$
zbar

a) $\begin{array}{llll}\mathrm{B} & . & \text { sehr schwer schmelzbar } \\ \mathrm{Al} & . & . & \mathbf{6 5 8}\end{array}$
b) Ga . . 30.15
$\mathrm{Jn} . . .154$
TI . . . 303.7
303.15
427
576.7

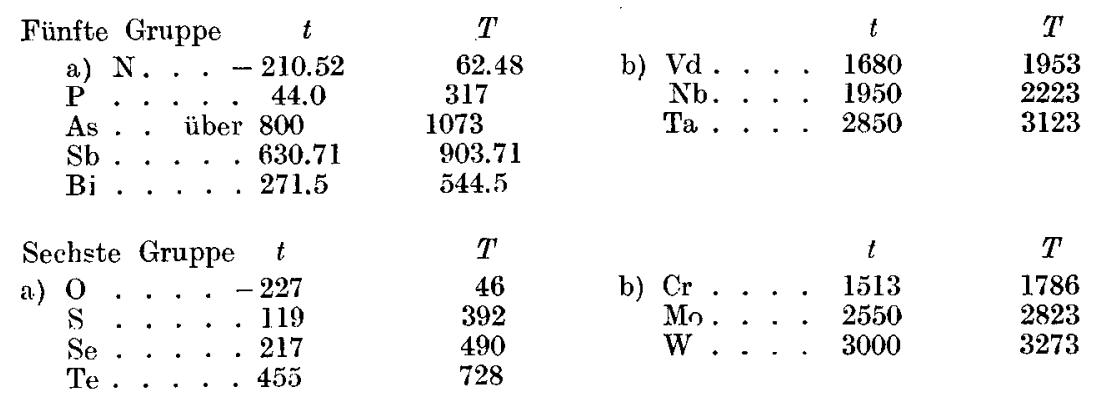




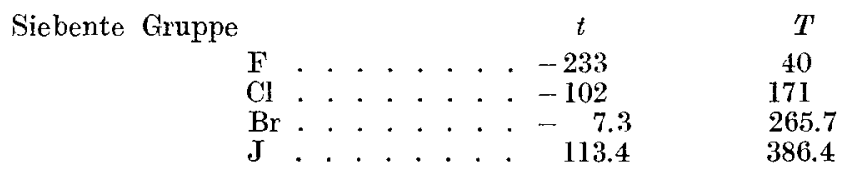

Schmelzpunktsregelmäßigkeiten sind deutlich vorhanden, doch treten auch mancherlei Unstetigkeiten auf. Innerhalb jeder Gruppe heben sich die Haupt- und Nebenreihe voneinander ab. Neigung zum Steigen der Schmelzpunkte mit wachsendem Atomgewicht besteht durchgängig bei den Metalloiden (analog wie bei den Siedepunkten) in Gruppe Null, Fünf a bei den ersten drei Gliedern, Sechs a und Sieben, während das Schmelzpunktsverhältnis von Kohlenstoff und Silicium unbestimmt bleibt. Für die metallischen Reihen läßt sich keine Grundlage für fallende oder sinkende Schmelzpunkte erkennen; man kann nur sagen, dab in den Metallreihen Eins a und Zwei b die Schmelzpunkte (ebenso wie die Siedepunkte) fallen, und daß in Gruppe Fünf a Steigen und Sinken der beiden Temperaturpunkte eine ähnliche Tendenz aufweisen; dagegen variieren die Schmelz- und Siedepunkte von Zinn und Blei in umgekehrter Richtung. Einzelne herausfallende Schmelzpunkte sind in der Zusammenstellung durch Sternchen bezeichnet.

Die Tatsache, daß dje Reihenfolge der Schmelz- und siedepunkte der Elemente im allgemeinen in gleicher Richtung verläuft, legt den Gedanken an eine bestimmte Abhängigkeit $\mathrm{z}$ wischen diesen beiden Größen nahe, und in der Tat ist von H. FrITz ${ }^{1}$ die Annahme ausgesprochen worden, daß das Verhältnis von Schmelzpunkt zu Siedepunkt in absoluter Zählung immer den Wert

$$
1: 1.2
$$

oder $1: 1.8=3 / 2 \cdot 1.2$

oder $1: 2.7=3 / 2 \cdot 1.8$

haben soll; doch ist diese Angabe zweifellos unhaltbar, und auch die einfachere Vorstellung, daß der Quotient der beiden Temperaturen innerhalb einer Gruppe konstant ist oder eine einfache Reihe bildet, zeigt vielerlei Unstetigkeiten, wenn auch ein gewisser Zu sammenhang unter den Quotienten nicht zu verkennen ist.

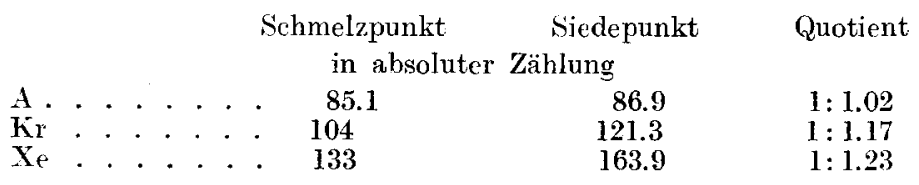

1 Monatshefte d. Chem. 13, 743. 


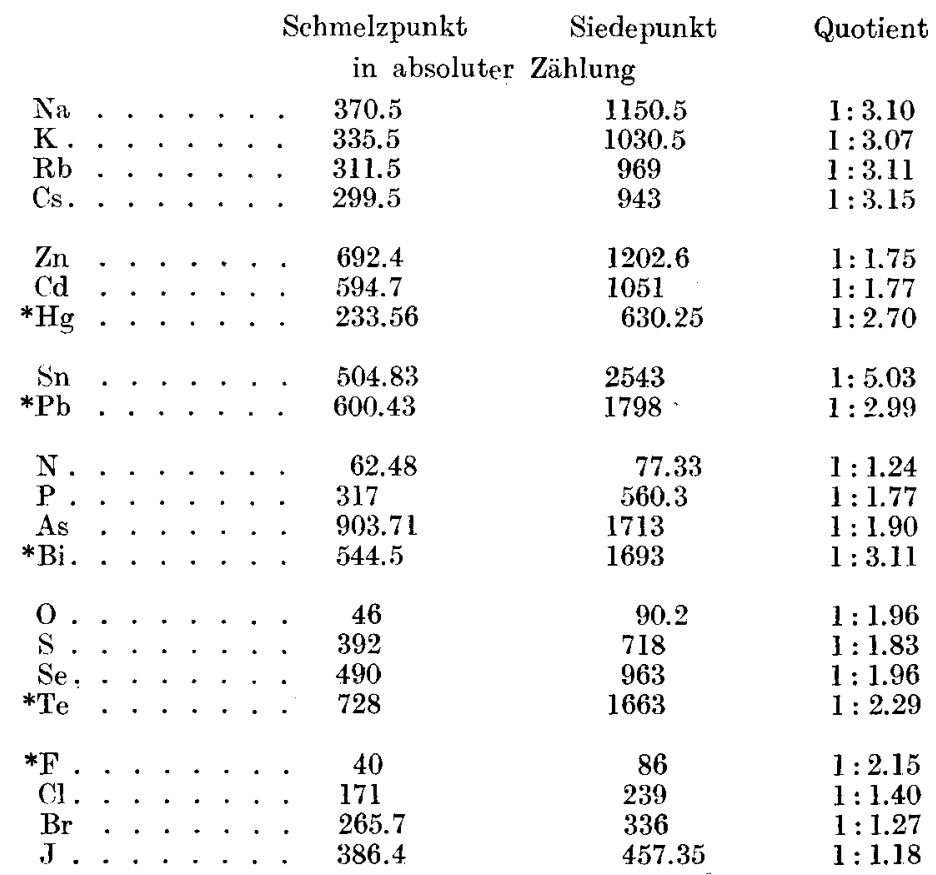

Infolge der auf Seite 6 besprochenen Regel von Guldbera, wonach der Siedepunkt etwa zwei drittel der kritischen Temperatur beträgt, ist es selbstverständlich, daß eine ähnliche Zusammengehörigkeit wie zwischen Siede- und Schmelzpunkt auch zwischen Schmelzpunkt und kritischer Temperatur besteht. Von ClankE ${ }^{1}$ ist die Forderung ausgesprochen worden, daß der Quotient aus kritischer und Schmelztemperatur in absoluter Zählung konstant sein soll. Leider ist unsere Kenntnis von den kritischen Temperaturen der Elemente so begrenzt, daß die Prüfung nur in zwei Reihen möglich ist, wo aber keine Konstanz, sondern einmal ein leicht steigender und einmal ein leicht fallender Gang erscheint.

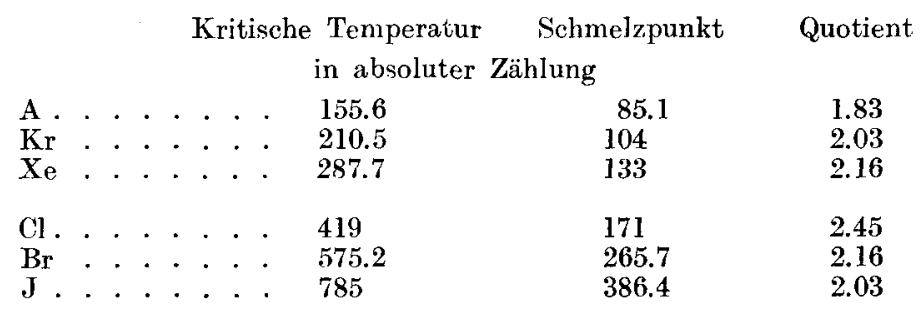

1 Amer. Chem. J. 18, 618. 
Um diese Clarkesche Beziehung auch für einige Verbindungen berechnen zu können, gebe ich die folgende Zusammenstellung, wo die Schmelzpunkte in gewöhnlicher $(t)$ und absoluter $(T)$ Zählung and die kritischen Temperaturen $\left(T_{k}\right)$ aufgeführt sind.

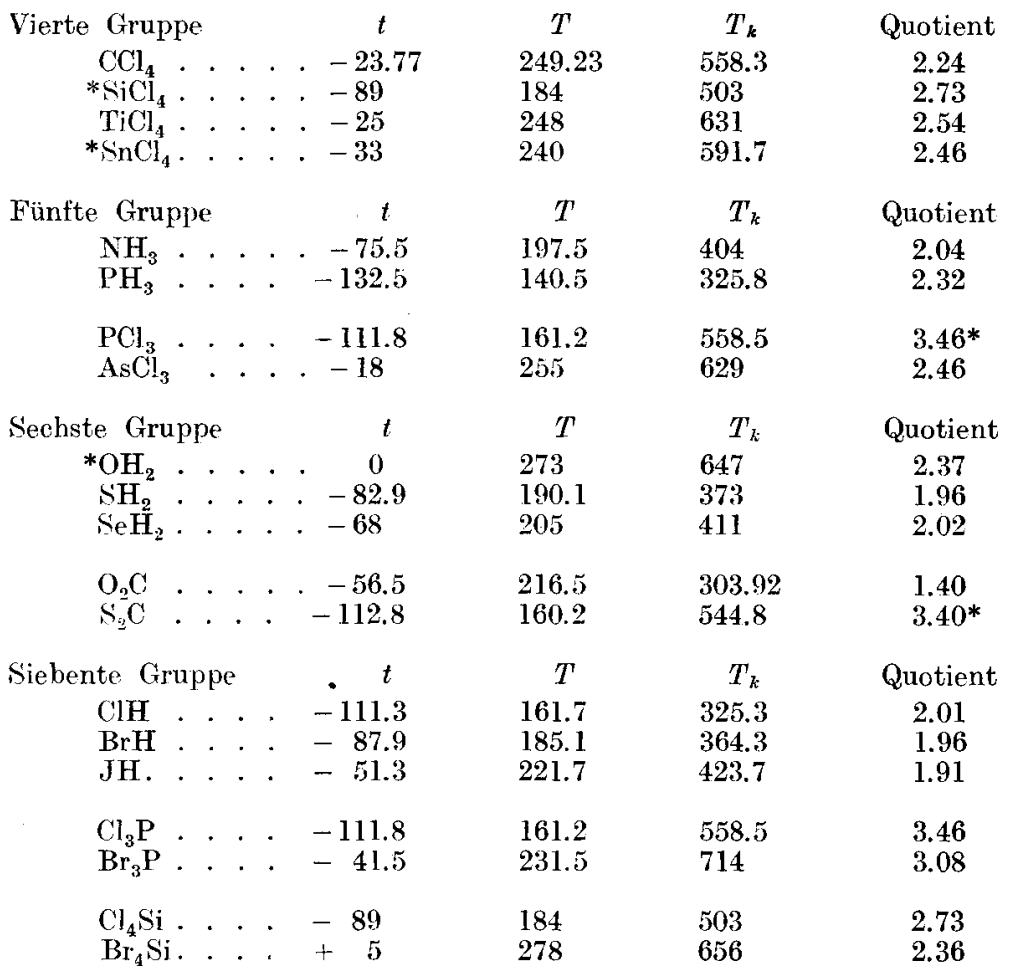

Die Siedepunkte und kritischen Temperaturen dieser Verbindungen zeigen im allgemeinen einen regelmäßigen Verlauf. In den meisten Fällen steigen beide Größen mit dem Molekelgewicht; ein Sinken der zwei Werte haben wir nur bei $\mathrm{NH}_{3}-\mathrm{PH}_{3}$, während bei $\mathrm{O}_{2} \mathrm{C}-\mathrm{S}_{2} \mathrm{C}$ die Siedepunkte fallen und die kritischen Temperaturen wachsen. Unstetigkeiten erscheinen bei den Chloriden der vierten Gruppe und in der sechsten Gruppe beim Wasser, welche Verbindungen entsprechend auch Anomalien bei den Siedepunkten aufweisen, ein Verhalten, das vielleicht auf besondere molekulare Bedingungen deutet.

Die berechneten CLARKE schen Quotienten liefern zweifellos einen gewissen Zusammenhang; nur in den Fällen $\mathrm{PCl}_{3}-\mathrm{AsCl}_{3}$ und $\mathrm{O}_{2} \mathrm{C}-\mathrm{S}_{2} \mathrm{C}$ versagt die Regel.

Den eben angeführten Schmelzpunkten möchte ich noch die 
einiger Metallverbindungen anreihen, die wiederum dartun, daß in den Gruppen des periodischen Systems regelmäßige Tendenzen vorhanden sind, daß aber zahlreiche Ausnahmen die Existenz einer eindeutigen Beziehung verhindern. Die Schmelzpunkte sind nur in gewöhnlicher Zählung angeführt.

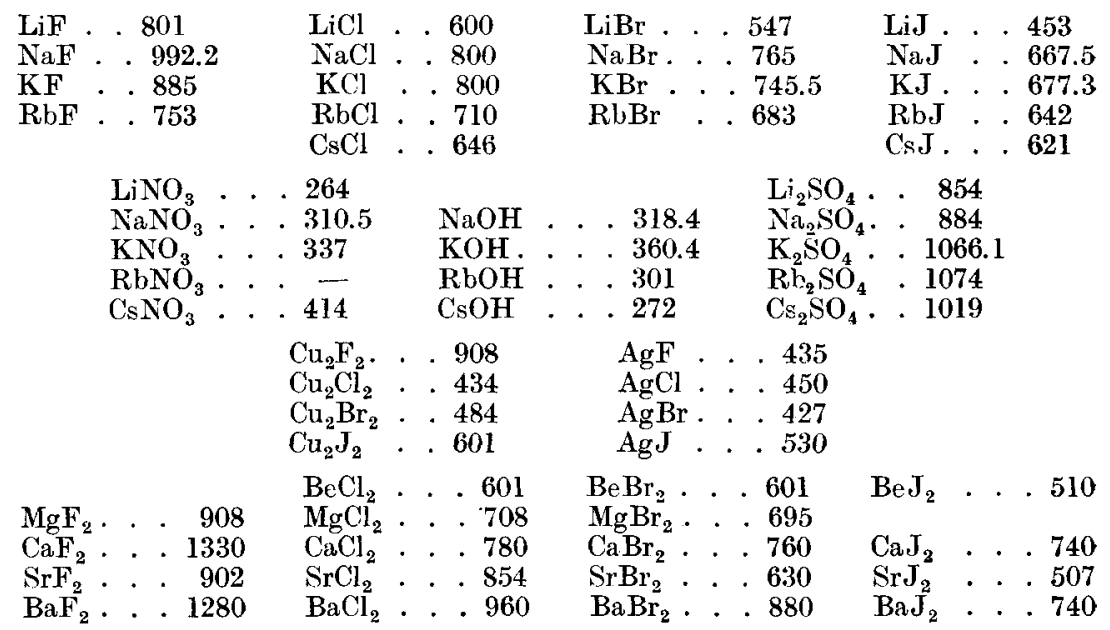

Eine sehr gute Regelmäßigkeit ergibt sich bei der Zusammenstellung der Schmelzwärmen von Elementen und Verbindungen. Ganz allgemein nimmt die Schmelzwärme (pro Kilogramm Sub$\operatorname{stanz)}$ mit wachsendem Atomgewicht $a b$. Erste Gruppe

Schmelzwärmen der Elemente.

$$
\begin{aligned}
& \mathrm{Na} \text {... . } 31.7 \mathrm{Cu} \text {. . . } 43 \\
& \mathrm{~K} . \ldots .15 .7 \quad \mathrm{Ag} \ldots 21.1
\end{aligned}
$$

Zweite Gruppe

$$
\begin{aligned}
& \mathrm{Zn} \text {. . . . } 28.1 \\
& \text { Cd . . . . } 13.7 \\
& \mathrm{Hg} \text {. . . } 2.84
\end{aligned}
$$

Siebente Gruppe

$\mathrm{Cl} \cdot . \cdot 22.96$

J. . . 11.7 (totale Schmelzwärme)

Schmelzwärmen von Verbindungen.

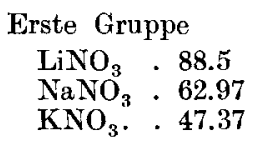

$$
\begin{aligned}
& \mathrm{NaOH} \cdot \cdot 40.0 \\
& \mathrm{KOH} \cdot \cdot 28.6 \\
& \mathrm{RbOH} \cdot \cdot 15.8 \\
& \mathrm{CsOH} \cdot \cdot 10.7
\end{aligned}
$$

Siebente Gruppe

$$
\begin{aligned}
& \mathrm{Cl}_{2} \mathrm{~Pb} \text {. . } 18.5 \\
& \mathrm{Br}_{2} \mathrm{~Pb} \text {. } 12.34 \\
& \mathrm{~J}_{2} \mathrm{~Pb} \text {. . } 11.50
\end{aligned}
$$

Vierte Gruppe

$\mathrm{Sn} . .14 .25$

$\mathrm{Pb} . . .5 .37$

\section{Achte Gruppe}

$\mathrm{Pd}$. . . . . 36.3

Pt:. $: 27.2$

Zweite Gruppe

${ }^{* \mathrm{SrCl}_{2}} \cdot 2.62{ }^{2} \mathrm{SbBr}_{3} \cdot 9.76$

$\mathrm{BaCl}_{2} \cdot 27.8$ 
Ausnahmen bilden nur $\mathrm{SrCl}_{2}$ und $\mathrm{sbBr}_{3}$, bei denen aber vielleicht Wassergehalt oder Zersetzlichkeit die Ursache der - zahlenmäßig geringen - Anomalie sein können.

Diese umgekehrte Proportionalität zwischen Atomgewicht und Schmelzwärme legt den Gedanken nahe, daß vielleicht gemäß dem Gesetz von 1)ulong und Petrit die Produkte von Atomgewicht und Schmelzwärme einen konstanten Wert annehmen; aber das ist nicht der Fall. Selbst innerhalb jeder Gruppe des periodischen Systems zeigen diese Produkte einen Gang, der mit steigendem Atomgewicht wächst oder fällt. Diese Produkte betragen abgerundet:

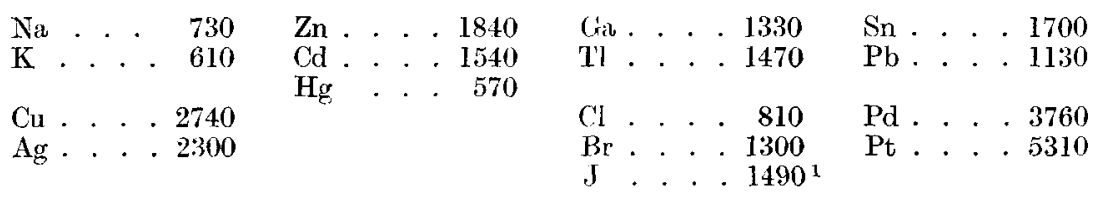

Bisher wurden die Schmelzpunkte der Elemente nur innerhalb der senkrecht stehenden Familien verglichen; die Betrachtung der Horizontalreihen liefert (Schmelzpunkte in absoluter Zählung):

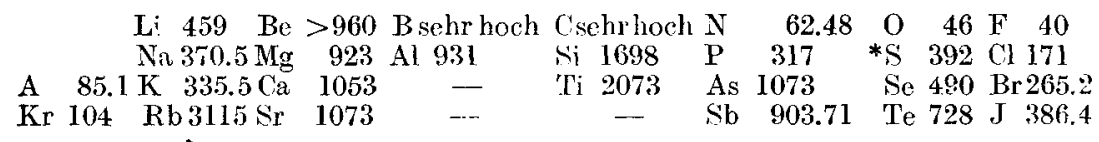

Es ist eine deutliche Periodizität vorhanden, indem die Schmelzpunkte von links anfangend steigen, in der Mitte ihr Maximum erreichen und dann wieder fallen; doch kommen auch hier Anomalien vor, wie die verkehrt laufenden Werte bei Phosphor und Schwefel zeigen.

Bei einem Vergleich anderer Eigenschaften der Elemente und homologer Verbindungen ergibt sich als allgemeine Schwierigkeit der Umstand, daß das Verhalten eines Stoffes erheblich von den äußeren Bedingungen, speziell der Temperatur abhängig ist, und daß die Auswahl einer Temperatur, wo die Stoffe in übereinstimmenden Zuständen vorliegen, Schwierigkeiten bereitet. Dazu kommt noch, $d a \beta$ die Eigenschaften der Stoffe meist nur bei irgendeiner, durch die Zufälligkeit der Versuchsanordnung gegebenen Temperatur bestimmt sind. Erfolgreich wird man erst dann in die besonderen Gesetzmäßigkeiten des periodischen Systems eindringen können, wenn diese Unzulänglichkeit beseitigt und das studierte Tatsachenmaterial unter wirklich vergleichbaren äußeren Umständen in

1 Nicht eigentlich vergleichbar, da hier die totale Schmelzwärme benutzt ist. 
weitestem Umfange bekannt ist. Für die großartige Einfachheit der vorhandenen Beziehungen spricht es, daß trotz aller Mängel viele Eigenschaften nach dem System sich direkt vergleichen lassen, und ich möchte diese RegelmäBigkeiten noch durch einige Tabellen hervorheben, die im allgemeinen bei der Anführung des periodischen Systems in den Lehrbüchern nicht immer betont werden.

Am günstigsten für den Vergleich steht es mit dem Brechungsvermögen, da man bei diesem durch die bekannten Formeln von Lorentz und Lorenz $\frac{n^{2}-1}{n^{2}+\frac{1}{2}} \cdot \frac{1}{d}$ oder Gladstone und Dale $\frac{n-1}{d}$ wo $n$ der Brechungsquotient und $d$ die Dichte bedeuten, eine Funktion von $n$ besitzt, die von der 'Temperatur unabhängig ist. GLadstone ${ }^{1}$ hat 1896 die Atomrefraktionen der Elemente zusammengestellt, indem er diese nach dem LandouTschen Gesetz der Additivität der Molekularrefraktion aus den Brechungen der Verbindungen berechnete. Seitdem ist aber eine ganze Zahl neuer Bestimmungen veröfentlicht worden, welche den Vergleich direkter Messungen gestatten und mit Auswahl von mir gesammelt sind. Unter $t$ ist bei dieser Aufzählung stets die Temperatur in gewöhnlicher Zählung, unter $\lambda$ die Wellenlänge, unter $d$ die Dichte und unter $n$ der Brechungsexponent angegeben.

Brechungsvermögen flüssiger Elemente.

\begin{tabular}{ccccccc} 
Fünfte Cruppe $t$ & \multicolumn{1}{c}{$t$} & $n$ & $d$ & $\frac{\mathrm{n}^{2}-1}{n^{2}+2} \cdot \frac{1}{d}$ & $\frac{n^{2}-1}{n^{2}+2} \cdot \frac{\text { Atomgew. }}{d}$ \\
N..-190 & 0.589 & 1.205 & 0.7851 & 0.167 & 2.33 \\
P. . & 44 & 0.656 & 2.050 & 1.814 & 0.284 & 8.83 \\
Sechste Gruppe & & & & & \\
O. . - -181 & 0.589 & 1.221 & 1.1181 & 0.126 & 2.01 \\
S. . . 110 & 0.589 & 1.929 & 1.8114 & 0.263 & 8.40 \\
Siebente Gruppe & & & & & \\
Cl. . . 14 & 0.589 & 1.367 & 1.4273 & 0.157 & 5.59 \\
Br. . . ? & 0.589 & 1.661 & 3.120 & 0.118 & 6.77
\end{tabular}

Mit steigendem Atomgewicht wachsen in jeder Reihe die Brechungskoeffizienten und die daraus berechneten, in der letzten Kolumne stehenden Atomrefraktionen.

Im allgemeinen trifft man das gleiche Verhalten auch bei flüssigen Verbindungen wieder, wo aber einzelne Unregelmäßigkeiten erscheinen, die durch Sternchen hervorgehoben sind. Ich habe mich im folgenden auf die Wiedergabe der Berechnungsexponenten beschränkt.

1 Proc. Roy. Soc. London 66, 140. 


\begin{tabular}{|c|c|c|c|c|c|c|c|c|}
\hline Vierte $G$ & Gruppe & & & & & $t$ & $\lambda$ & $n$ \\
\hline & $\mathrm{CCl}_{4}$ & . & . . & & & 15 & 0.434 & 1.483 \\
\hline & * $\mathrm{SiCl}_{4}$ & . & . . & & & 20 & 0.434 & 1.424 \\
\hline & $\mathrm{SnCl}_{9}$ & 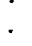 & & 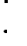 & & 20 & 0.434 & 1.537 \\
\hline Fïnfte & Gruppe & & & & & $t$ & $\lambda$ & $n$ \\
\hline & $\mathrm{NH}_{3}$ & 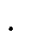 & * & & & 16.5 & 0.589 & 1.325 \\
\hline & ${ }^{*} \mathrm{PH}_{3}$. & 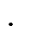 & . & . & & 17.5 & 0.589 & 1.317 \\
\hline & $\mathrm{PCl}_{3}$. & . & . . & . & & 14 & 0.434 & 1.535 \\
\hline & $\mathrm{AsCl}_{3}$ & $\cdot$ & . . & & & 14 & 0.434 & 1.625 \\
\hline Sechste & Gruppe & & & & & $t$ & $\lambda$ & $n$ \\
\hline & $\mathrm{SO}_{2} \mathrm{Cl}_{2}$ & . & . . & . & & 12.4 & 0.589 & 1.444 \\
\hline & & - & . & & & & 0.589 & 1.524 \\
\hline Siebente & e Gruppe & & & & & $t$ & $\lambda$ & $n$ \\
\hline & $\mathrm{Cl}_{3} \mathrm{~B}$ & ${ }^{\circ}$ & . & & & 5.7 & 0.486 & 1.428 \\
\hline & $\mathrm{Br}_{3} \mathrm{~B}$ & 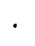 & - & & & 6.3 & 0.486 & 1.553 \\
\hline & $\mathrm{Cl}_{3} \mathrm{P}$. & . & $\cdot$ & & & 14 & 0.434 & 1.535 \\
\hline & $\mathrm{Br}_{3} \mathrm{P}$ & . & 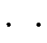 & & & 25 & 0.434 & 1.733 \\
\hline & ClH & . & . & . & & 10.5 & 0.589 & 1.254 \\
\hline & $\mathrm{BrH}$ & . & . & $\cdot$ & & 10 & 0.589 & 1.325 \\
\hline & $\mathrm{JH}$ & 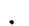 & . & . & & 12 & 0.589 & 1.466 \\
\hline
\end{tabular}

Abweichungen von dem gleichzeitigen Steigen der Atomgewichte und Berechnungsquotienten finden sich in der vierten Gruppe und in der fünften bei Ammoniak und Phosphorwasserstoff, wo vorher Anomalien auch für andere Eigenschaften aufgefunden worden sind.

Brechungsexponenten von gasförmigen Elementen bei $0^{0}$ gegen den luftleeren Raum.

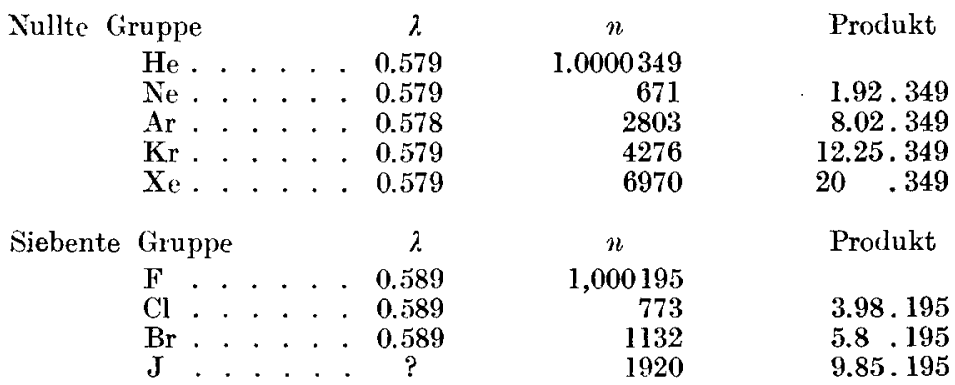

Auch hier entspricht innerhalb jeder Gruppe dem Steigen des Atomgewichts eine Vergrößerung des Brechungsexponenten.

Von Cuthiertson und Prideaux ${ }^{1}$ stammt die Angabe, daß bei Elementen derselben Gruppe des periodischen Systems die Werte $n-1$ in einem ganzzahligen Verhältnis zueinander stehen.

1 Proc. Roy. Soc. London, Ser. A. 76, 426. 
Eine sehr angenäherte Beziehung derart ist auch in der Tat vorhanden, wie die angegebenen Produkte erweisen.

Auch bei gasförmigen Verbindungen steigen die Brechungsexponenten mit den Atomgewichten $(t=0)$ innerhalb jeder Gruppe.

\begin{tabular}{|c|c|c|c|c|c|c|c|c|c|c|c|c|}
\hline Fünfte 0 & Gruppe & & & & & & & & & & $\lambda$ & $n$ \\
\hline & $\mathrm{NH}_{3}$ & & & & . & & . & & & & 0.589 & 1.000379 \\
\hline & $\mathrm{PH}_{3}$ & & . & - & . & . & . & . & . . & . & ? & 789 \\
\hline Sechste & Gruppe & & & & & & & & & & $\lambda$ & $n$ \\
\hline & $\mathrm{OH}_{2}$ & & & & $\cdot$ & & & . & & & 0.589 & 1.000249 \\
\hline & $\mathrm{SH}_{2} \cdot$ & & t & & . & & $\cdot$ & . & - . & - & 0.589 & 623 \\
\hline & $\mathrm{O}_{2} \mathrm{C}$ & & . & & $\cdot$ & & . & . & . & . & 0.589 & 1.000449 \\
\hline & $\mathrm{S}_{2} \mathrm{C}$ & & & - & . & . & . & & . & . & 0.589 & 1478 \\
\hline Siebente & Grupp & & & & & & & & & & $\lambda$ & $n$ \\
\hline & $\mathrm{ClH}$ & & & & . & & . & . & - . & & 0.589 & 1.000447 \\
\hline & $\mathrm{BrH}$ & & & & $\cdot$ & & . & . & . & . & 0.589 & 573 \\
\hline & JH . & & & & . & & . & . & - . & & 0.589 & 911 \\
\hline & $\mathrm{ClCH}_{3}$ & & & • & . & . & . & . & . & • & 0.589 & 1.000870 \\
\hline & $\mathrm{BrCH}_{3}$ & & & & & . & & & . & . & 0.589 & 964 \\
\hline & $\mathrm{JCH}_{3}$ & & & & & & & & . & . & 0.589 & 1265 \\
\hline
\end{tabular}

Dieselbe Regelmäßigkeit zeigt sich auch bei den folgenden Werten für Gase bei $0^{0}$, wo die Größe $n_{0}$ aus dem beobachteten $n$ nach der Gleichung

$$
\frac{n_{0}-1}{n-2}=\frac{\text { normale Dichte }}{\text { beobachtete Dampfdichte }}
$$

berechnet worden ist. Die Wellenlänge beträgt immer 0.589 .

\begin{tabular}{|c|c|c|c|}
\hline Ziveite & Gruppe & ünfte Gruppe & Sechste Gruppe \\
\hline & $n_{0}$ & $n_{0}$ & \\
\hline & 1.002060 & 1.001212 & 1.001111 \\
\hline $\begin{array}{l}\mathrm{Cd} \\
{ }^{\mathrm{H} g}\end{array}$ & $\begin{array}{l}1.002675 \\
1.001866\end{array}$ & 1.001550 & $\begin{array}{l}1.001565 \\
1.002495\end{array}$ \\
\hline
\end{tabular}

Hierbei fällt das Quecksilber heraus.

Den Schluß mögen einige Berechnungsquotienten der D-Linie von Lösungen machen, die in einer Konzentration von $10 \%$ und bei $17.5^{\circ}$ untersucht worden sind.

\begin{tabular}{|c|c|c|c|c|c|}
\hline \multirow{3}{*}{$\begin{array}{l}\mathrm{NaCl} . \\
\mathrm{KCl} .\end{array}$} & $n$ & \multicolumn{2}{|r|}{$n$} & & $n$ \\
\hline & 1.34963 & $\mathrm{NaBr}$ & . . 1.34672 & $\mathrm{Na} \mathbf{J}$ & 1.34758 \\
\hline & $1.34 \tilde{5} 98$ & $\mathrm{KBr}$ & . . 1.34480 & KJ & . . 1.34612 \\
\hline & $n$ & & $n$ & & $n$ \\
\hline $\mathrm{NaNo}_{3}$ & 1.34393 & $\mathrm{NaOH}$ & . . 1.35755 & $\mathrm{MgCl}_{2}$ & 1.35703 \\
\hline $\mathrm{KNO}_{3}$ & 1.34212 & $\mathrm{KOH}$. & . . 1.35151 & $\mathrm{CaCl}_{2}$. & 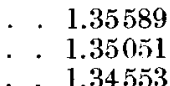 \\
\hline Z. an & allg. $\mathrm{Ch}$ & & & & 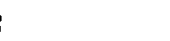 \\
\hline
\end{tabular}


Mit steigendem Atomgewicht sinken die $n$-Werte bei den Lösungen, also gerade umgekehrt wie es bei den reinen Flüssigkeiten und Gasen der Fall war.

In naher Beziehung zu den Brechungsexponenten stehen die Dielektrizitätskonstanten, für deren Regelmäßigkeiten verschiedene Reihen angeführt werden können.

Von Elementgruppen kommen die nachstehenden Dielektrizitätskonstanten $\varepsilon$ in Betracht, die mit steigendem Atomgewicht wachsen.

\begin{tabular}{|c|c|c|c|c|c|}
\hline Sechste & Gruppe & $\lambda$ & $t$ & $\varepsilon$ & Aggregatzustand \\
\hline $\mathrm{O}$ & ... & $\infty$ & -182 & 1.491 & flüssig \\
\hline s & . & $\infty$ & nahe beim Siedep. & 3.42 & flüssig \\
\hline Se & . & $\infty$ & $?$ & 6.13 & glasig \\
\hline Siebente & Gruppe & $\lambda$ & $t$ & $\varepsilon$ & Aggregatzustand \\
\hline $\mathrm{Cl}$ & . . & $\infty$ & -60 & 2.150 & flüssig \\
\hline $\mathrm{Br}$ & . . & $\infty$ & 1 & 4.6 & flüssig \\
\hline
\end{tabular}

Bei Verbindungen findet mit steigendem Atomgewicht meist ein Sinken, manchmal aber auch ein Steigen der Dielektrizitätskonstanten statt.

\begin{tabular}{|c|c|c|c|c|c|c|}
\hline Erste Gruppe & & & & $\lambda$ & $\varepsilon$ & Aggregatzustand \\
\hline $\mathrm{NaCl}$ & & & . & $\infty$ & 6.29 & fest \\
\hline $\mathrm{KCl}$ & & & . . & $\infty$ & 4.94 & fest \\
\hline $\mathrm{NaNO}_{3}$ & & & . & $\infty$ & 5.18 & fest \\
\hline $\mathrm{KNO}_{3}$ & & & . $\cdot$ & $\infty$ & 2.56 & fest \\
\hline Zweite Gruppe & & & $\lambda$ & $t$ & $\varepsilon$ & Aggregatzustand \\
\hline $\mathrm{SrSO}_{4}$ & & & 75 & - & 11.3 & fest \\
\hline $\mathrm{BaSO}_{4}$ & & & 75 & 一 & 10.2 & fest \\
\hline Vierte Gruppe & & & $\lambda$ & $t$ & $\varepsilon$ & Aggregatzustand \\
\hline $\mathrm{CCl}_{4}$ & & & 73 & 17 & 2.18 & flüssig \\
\hline $\mathrm{SiCl}_{4}$ & & & 80 & 16 & 2.40 & flüssig \\
\hline $\mathrm{snCl}_{4}$ & & & 84 & 22 & 3.2 & flüssig \\
\hline Fünfte Gruppe & & & $\lambda$ & $t$ & $\varepsilon$ & Aggregatzustand \\
\hline $\mathrm{NH}_{3} \cdot$ & & & 72 & $-\mathbf{5 0}$ & 22.7 & flüssig \\
\hline $\mathrm{PH}_{3}^{\circ}$ & & & 72 & -50 & 2.6 & flüssig \\
\hline $\mathrm{AsH}_{3}$ & & & 72 & -50 & 2.58 & flüssig \\
\hline $\mathrm{SbH}_{3}$. & & & 72 & -50 & 2.58 & flüssig \\
\hline Sechste Gruppe & & & 2. & $t$ & $\varepsilon$ & Aggregatzustand \\
\hline $\mathrm{OH}_{2}$ & & & $\infty$ & 18 & 81.1 & flüssig \\
\hline $\mathrm{SH}_{2}$ & & & $\infty$ & 10 & 5.93 & flüssig \\
\hline $\mathrm{SO}_{2} \mathrm{Cl}_{2}$. & & & 73 & 25 & 8.5 & flüsssig \\
\hline $\mathrm{CrO}_{2} \mathrm{Cl}_{2}$. & & & 73 & 20 & 2.6 & flüssig \\
\hline
\end{tabular}




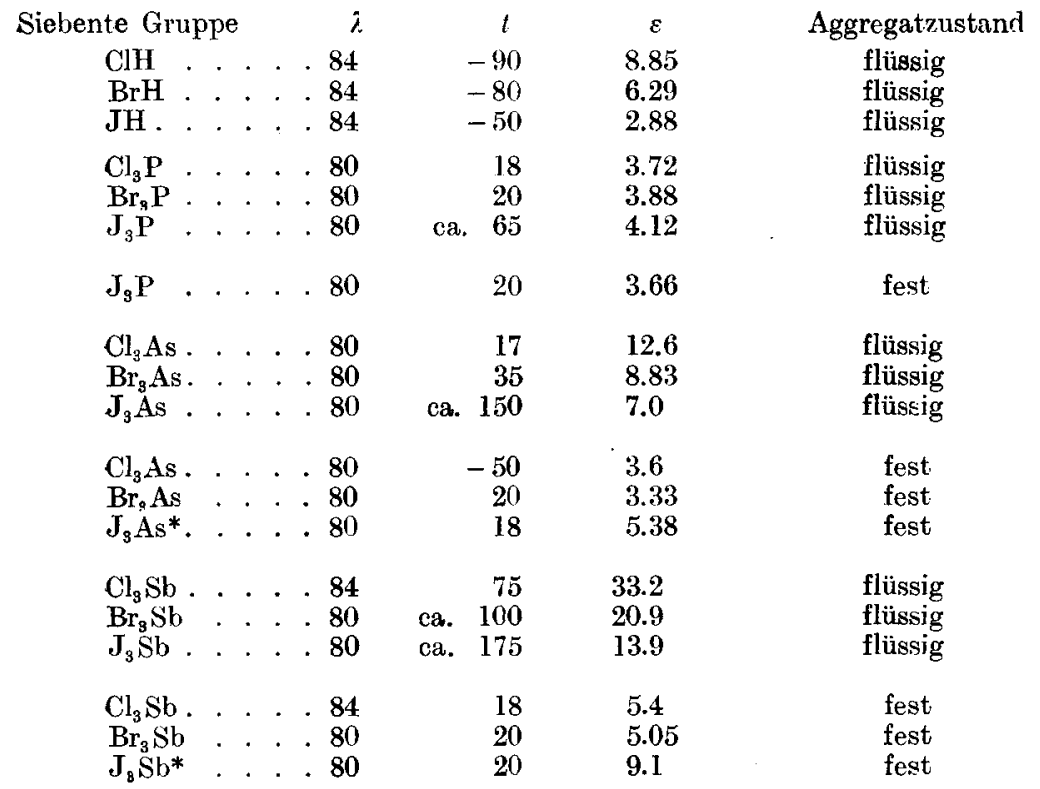

Werden die Haloide von Phosphor, Arsen und Antimon in Abwandlung dieser drei Elemente zusammengestellt, so ist die Reihenfolge:

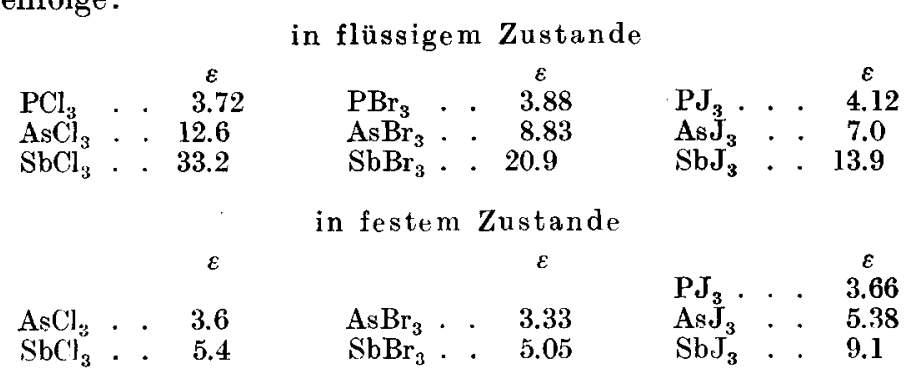

Ausnahmen bilden nur die festen Formen von $\mathrm{AsJ}_{3}$ und $\mathrm{SbJ}_{3}$ an den durch Sternchen bezeichneten Stellen.

Eine sehr gute Regelmäßigkeit liefern weiterhin die Diffusionskoeffizienten, welche Metalle, die zu $0.1 \%$ igem Amalgam gelöst sind, gegen Quecksilber zeigen; die entsprechende Tabelle ist ohne weiteres verständlich.

Erste Gruppe

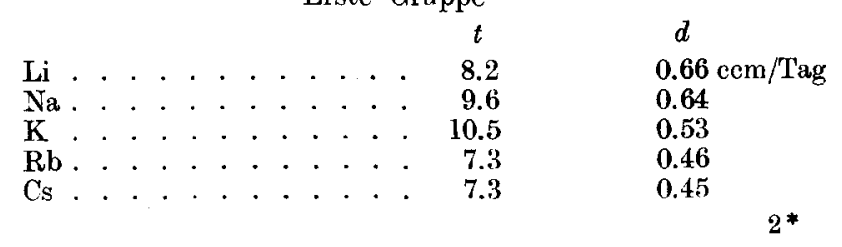




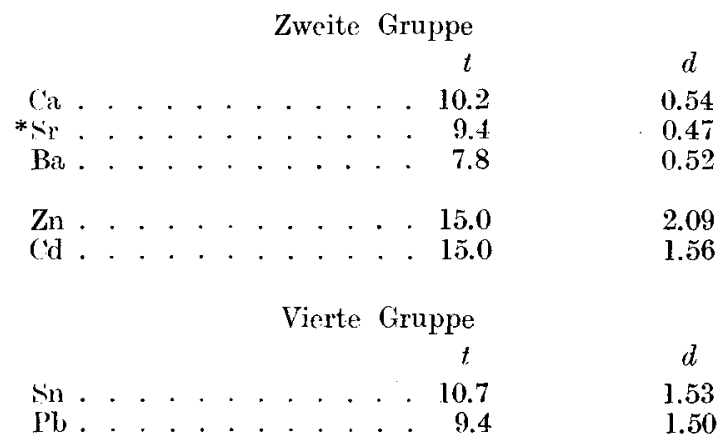

Die Diffusionskoeffizienten nehmen mit steigendem Atomgewicht $a b$, nur bei Strontium-Barium findet sich eine Anomalie. So regelmäßig der Gang der Diffusionskoeffizienten erscheint, so ist andererseits hervorzuheben, da $B$ diese Koeffizienten keineswegs vom Atomgewicht allein abhängen, wie beispielsweise die Tatsache erweist, daB Kalium mit dem Atomgewicht 39 und Baryum mit dem Atomgewicht 137 nahezu den gleichen Diffusionskoeffizienten besitzen; wahrscheinlich hat hier die Bildung von Metall-Quecksilberverbindungen ${ }^{1}$ wesentliche Bedeutung.

Über I,öslichkeitsregelmäßigkeiten von Salzen nach dem periodischen System liegt ein großes Tatsachenmaterial vor; dagegen sind naturgemäß Angaben über die Löslichkeit der Elemente selbst nur wenig bekannt. Im großen ganzen kann es sich dabei nur um die Absorptionskoeffizienten gasförmiger Elemente handeln, von denen mir folgende Werte zur Verfügung stehen:

Absorptionskoeffizienten gasförmiger Elemente in Wasser

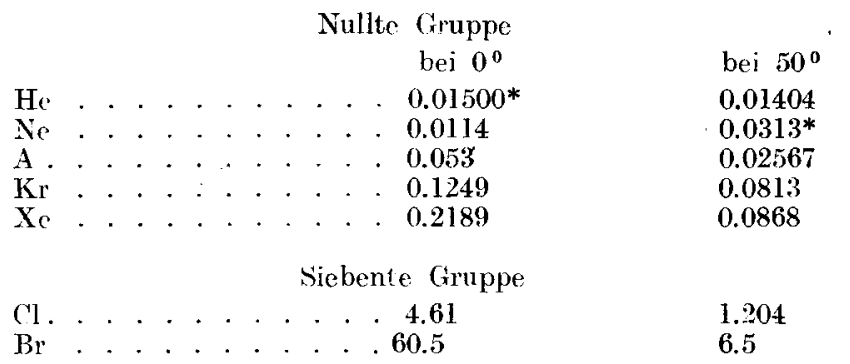

J)ie Absorptionskoeffizienten steigen mit dem Atomgewicht, doch tritt eine Unstetigkeit bei Helium oder Neon auf. 1)a die Löslichkeit des Neons - entgegen dem Verhalten der meisten Gase -

1 Beckmann und Liesche, Z. ahorg. Chem. 89, 171. 
mit wachsender Temperatur zunimmt, liegt die erwähnte Anomalie bei verschiedenen Temperaturen entweder bei diesem oder beim Helium.

Daß sich schließlich auch Eigenschaften, die dem Gedankenkreise des Chemikers im allgemeinen ferner liegen, nach den Atomgewichten ordnen lassen, ist durch das Beispiel der Schallgeschwindigkeiten $c$ bekannt, wofür die nachstehenden Werte anzugeben sind: Frste Gruppe

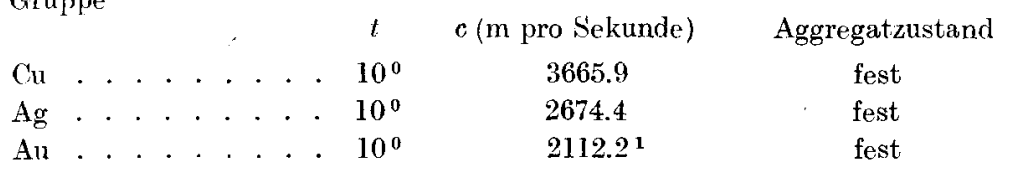

Zweite Gruppe

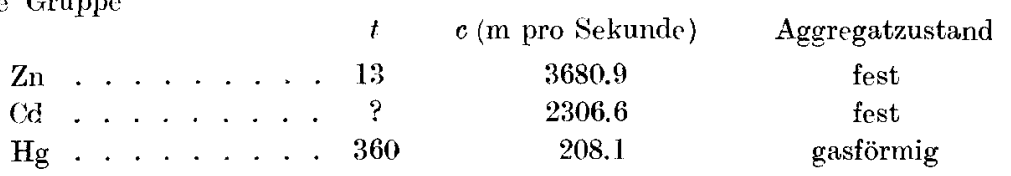

Vierte Gruppe

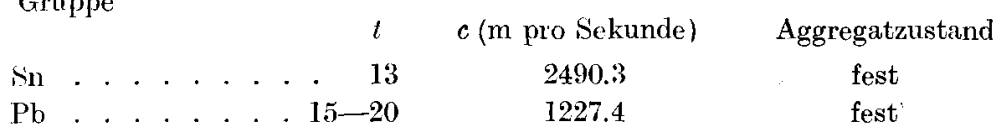

Siebente Gruppe

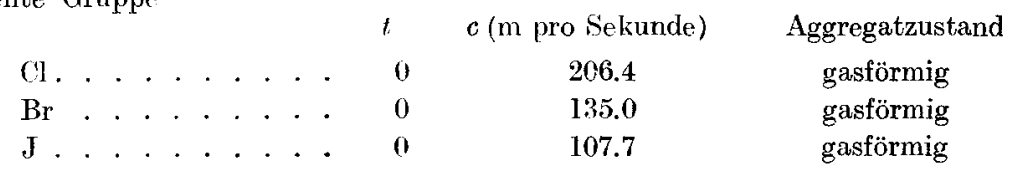

Die Schallgeschwindigkeiten sinken mit wachsendem Atomgewicht und ebenso mit steigenden Molekelgewichten bei den analogen Verbindungen:

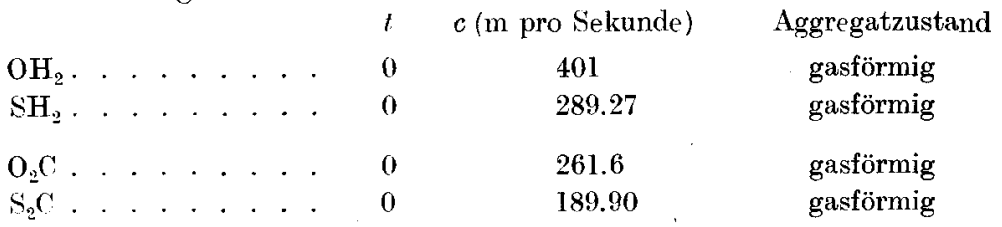

Nach Fritz ${ }^{2}$ soll das Produkt aus Atomgewicht und Schallgeschwindigkeit konstant sein, und das trifft auch in der Tat für die folgenden festen Elemente annähernd zu:

1 Der Wert schwankt nach der mechanischen Vorgeschichte des Metalls.

2 Monatsh. d. Chem. 13, 743. 
22 W. Herz. Zur Kenntnis einiger phys. Eigenschaften von Elementen usw.

$$
\begin{aligned}
& \mathrm{Cu} \text {. . . 3665.9. 63.57 }=233500 \\
& \mathrm{Ag} \text {. . . . } 2674.4 \cdot 107.88=288000 \\
& \mathrm{Zn} \text {. . . . 3680.9.65.37 }=241000 \\
& \text { Cd . . . 2 2306.6 . } 112.40=260000 \\
& \text { Sn . . . 2490.3 . 119.0 }=296000 \\
& \mathrm{~Pb} \text {. . . . 1227.4.207.1 }=253500
\end{aligned}
$$

Bei den gasförmigen Elementen treten andere Werte auf, die ebenso wie bei den gasförmigen Verbindungen mit wachsendem Atom- oder Molekelgewicht steigen.

Pharmazeulisches Institut der Universität Breslau, 11. Oktober 1915.

Bei der Redaktion eingegangen am 13. Oktober 1915. 